\title{
CONTINUITY IS AN ADJOINT FUNCTOR
}

\author{
EDWARD S. LETZTER
}

1. INTRODUCTION. The emergence of category theory, introduced by S. Eilenberg and S. Mac Lane in the 1940s (cf. [2]), was among the most important mathematical developments of the twentieth century. The profound impact of the theory continues to this day, and categorical methods are currently used, for example, in algebra, geometry, topology, mathematical physics, logic, and theoretical computer science. (Hints of this breadth can be found, e.g., in [1], [6], and [7]).

A category is comprised of objects and morphisms between objects. Standard elementary examples include $\mathcal{S}$ et, where the objects are sets and the morphisms are set functions; $\mathcal{G r p}$, where the objects are groups and the morphisms are group homomorphisms; and $\mathcal{T} o p$, where the objects are topological spaces and the morphisms are continuous functions.

Relationships among different categories are established via functors between them. A pair of adjoint functors, as formulated in 1958 by D. M. Kan [3], determines a particularly close tie between two categories. Adjoint functors are essential tools in category theory, and their introduction was a significant milestone in its development.

While it is commonly held that adjoint functors "occur almost everywhere" 6, p. 107]), at least in many areas of mathematics, the typical first examples presented to students may not immediately reveal the fundamental importance of the ideas involved. (One such typical example, a left adjoint to a "forgetful functor," is described at the end of the brief review provided in the next section.)

Our aim in this note, then, is to illustrate how a natural example of adjoint functors can be "found" in the definition of a continuous map between topological spaces. In particular, we show, for a set function $\varphi: X \rightarrow Y$ between topological spaces $X$ and $Y$, that $\varphi$ is continuous if and only if certain naturally arising functors are adjoint.

Remarks. The main result presented in this note was recorded in a more abstract, and apparently more obscure, setting in [5]. Moreover, in noncommutative algebraic geometry, certain adjoint functor pairs serve as morphisms between (not explicitly defined) noncommutative spaces. (This approach follows [8] and [10]; see also [9].)

The reader is referred for example to [4] for an accessible general introduction to category theory and its history.

2. A BRIEF RREVIEW. As mentioned above, a category $\mathcal{C}$ consists of objects and morphisms between objects. The morphisms in $\mathcal{C}$ from an object $A$ to an object

Date: January 1, 2014. 
$B$ comprise a set denoted $\operatorname{Hom}_{\mathcal{C}}(A, B)$. A morphism in $\mathcal{C}$ from $A$ to $B$ is also referred to as a $\mathcal{C}$-morphism and denoted $A \rightarrow B$.

These morphisms must satisfy the following conditions:

(1) For each pair of $\mathcal{C}$-morphisms $j: D \rightarrow E$ and $k: E \rightarrow F$, there is a composition morphism $k \circ j: D \rightarrow F$, such that

$$
\ell \circ(k \circ j) \text { and }(\ell \circ k) \circ j
$$

produce the same morphism $D \rightarrow G$, for all $\mathcal{C}$-morphisms $j: D \rightarrow E, k: E \rightarrow F$, and $\ell: F \rightarrow G$.

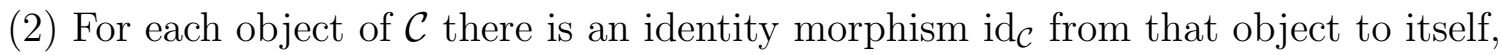
such that the compositions

$$
A \stackrel{\text { id }}{\longrightarrow} A \stackrel{f}{\longrightarrow} B \text { and } A \stackrel{f}{\longrightarrow} B \stackrel{\text { id }_{\mathcal{C}}}{\longrightarrow} B
$$

are both equal to $f$, for all $\mathcal{C}$-morphisms $f: A \rightarrow B$.

Adjoint Functors. Let $\mathcal{C}$ and $\mathcal{D}$ be categories. A (covariant) functor $\Phi: \mathcal{C} \rightarrow \mathcal{D}$ assigns to each object $A$ of $\mathcal{C}$ an object $\Phi(A)$ of $\mathcal{D}$, and to each $\mathcal{C}$-morphism $f: A \rightarrow B$ a $\mathcal{D}$-morphism

$$
\Phi(f): \Phi(A) \rightarrow \Phi(B)
$$

such that

$$
\Phi\left(\operatorname{id}_{\mathcal{C}}\right)=\operatorname{id}_{\mathcal{D}} \quad \text { and } \quad \Phi(\ell \circ k)=\Phi(\ell) \circ \Phi(k),
$$

for all $\mathcal{C}$-morphisms $k: E \rightarrow F$ and $\ell: F \rightarrow G$.

Now consider a pair of functors

$$
\Phi: \mathcal{C} \rightarrow \mathcal{D} \text { and } \Psi: \mathcal{D} \rightarrow \mathcal{C} .
$$

Also suppose, for all objects $L$ of $\mathcal{C}$ and $M$ of $\mathcal{D}$, that there exists a bijective function

$$
\operatorname{Hom}_{\mathcal{D}}(\Phi(L), M) \stackrel{\beta}{\longrightarrow} \operatorname{Hom}_{\mathcal{C}}(L, \Psi(M)),
$$

assigning to each $\mathcal{D}$-morphism

$$
r: \Phi(L) \rightarrow M
$$

a $\mathcal{C}$-morphism

$$
\beta(r): L \rightarrow \Psi(M)
$$

We then say that $(\Phi, \Psi)$ is an adjoint pair provided

$$
\beta(t \circ r)=\Psi(t) \circ \beta(r) \text { and } \beta(r \circ \Phi(s))=\beta(r) \circ s,
$$

for all $\mathcal{C}$-morphisms $s: L^{\prime} \rightarrow L$ and all $\mathcal{D}$-morphisms $t: M \rightarrow M^{\prime}$. We indicate that the bijection $\beta$ satisfies the two conditions in $(*)$ by saying that $\beta$ is natural in $L$ and $M$.

Example. The following is a standard first example of an adjoint pair: Let $\mathbb{F}$ be a field, and let $\mathcal{V} e c_{\mathbb{F}}$ denote the category whose objects are $\mathbb{F}$-vector spaces and whose morphisms are $\mathbb{F}$-linear transformations. Let $\Psi: \mathcal{V} e c_{\mathbb{F}} \rightarrow \mathcal{S}$ et be the "forgetful 
functor," assigning to each vector space its underlying set of vectors, and assigning to each linear transformation its underlying set function. Let $\Phi: \mathcal{S e t} \rightarrow \mathcal{V} e c_{\mathbb{F}}$ be the functor assigning to each set $S$ the $\mathbb{F}$-vector space $\mathbb{F} S$ with basis $S$, and assigning to each set map $S \rightarrow S^{\prime}$ the $\mathbb{F}$-linear extension $\mathbb{F} S \rightarrow \mathbb{F} S^{\prime}$. Then $(\Phi, \Psi)$ is an adjoint pair. (Details and analogous examples can be found, e.g., in Chapter IV of [6].)

3. CONTINUITY VIA ADJOINT PAIRS. For the remainder, let $X$ and $Y$ be topological spaces, and let $\varphi: X \rightarrow Y$ be a set function. Recall that $\varphi$ will be continuous if and only if $\varphi^{-1}(V)$ is closed in $X$ for all closed subsets $V$ of $Y$.

The category of closed subsets of a topological space. Define $\operatorname{Closed}(X)$ to be the category whose objects are closed subsets of $X$ and whose morphisms are described as follows: Let $U$ and $U^{\prime}$ be closed subsets of $X$. If $U$ is a subset of $U^{\prime}$ then there is exactly one morphism, the inclusion map, from $U$ to $U^{\prime}$. If $U$ is not a subset of $U^{\prime}$ then the set of morphisms from $U$ to $U^{\prime}$ is empty. Similarly define $\operatorname{Closed}(Y)$.

An adjointness criterion. Adjoint pairs of functors between the categories $\mathcal{C l o s e d}(X)$ and $\mathcal{C}$ losed $(Y)$ can be described in a particularly simple way, as follows: Suppose that

$$
\Phi: \mathcal{C l o s e d}(X) \rightarrow \mathcal{C} \text { losed }(Y) \text { and } \Psi: \mathcal{C} \text { losed }(Y) \rightarrow \mathcal{C} \text { losed }(X)
$$

are functors. Then for $U$ in $\mathcal{C l o s e d}(X)$ and $V$ in $\mathcal{C l o s e d}(Y)$, there exists a bijection

$$
\operatorname{Hom}_{C l o s e d(Y)}(\Phi(U), V) \stackrel{\beta}{\longrightarrow} \operatorname{Hom}_{C l o s e d}(X)(U, \Psi(V))
$$

exactly when one (and only one) of the following two cases holds:

Case 1: Both $\Phi(U) \subseteq V$ and $V \subseteq \Psi(V)$

Case 2: Both $\Phi(U) \nsubseteq V$ and $U \nsubseteq \Psi(V)$

It is not hard to verify that if a bijection $\beta$ as above does exist, then it must be natural in $U$ and $V$, in the sense of $(*)$. (Also note that if $\beta$ exists it must be unique.) We deduce that $(\Phi, \Psi)$ is an adjoint pair exactly when the statement

$$
\Phi(U) \subseteq V \text { if and only if } U \subseteq \Psi(V)
$$

holds true for all $U$ in $\mathcal{C}$ losed $(X)$ and $V$ in $\mathcal{C l o s e d}(Y)$.

From a function to a pair of functors. Consider the assignments

$$
T_{\varphi}: \mathcal{C l o s e d}(X) \longrightarrow \mathcal{C} \text { losed }(Y), \quad U \longmapsto \overline{\varphi(U)},
$$

and

$$
T^{\varphi}: \mathcal{C l o s e d}(Y) \longrightarrow \mathcal{C} \text { losed }(X), \quad V \longmapsto \overline{\varphi^{-1}(V)},
$$

where $\bar{S}$ denotes the closure of an arbitrary subset of $X$ or $Y$. It is straightforward to check that $T_{\varphi}$ and $T^{\varphi}$ are functors.

Our aim now is to prove:

Theorem. The function $\varphi$ is continuous if and only if $\left(T_{\varphi}, T^{\varphi}\right)$ is an adjoint pair. 
Proof. To start, we claim that the following four conditions are equivalent, for all $U$ in $\mathcal{C l o s e d}(X)$ and $V$ in $\mathcal{C l o s e d}(Y)$ :

(1) $\left(T_{\varphi}, T^{\varphi}\right)$ is an adjoint pair.

(2) $T_{\varphi}(U) \subseteq V$ if and only if $U \subseteq T^{\varphi}(V)$.

(3) $\overline{\varphi(U)} \subseteq V$ if and only if $U \subseteq \overline{\varphi^{-1}(V)}$.

(4) $\varphi(U) \subseteq V$ if and only if $U \subseteq \overline{\varphi^{-1}(V)}$.

The equivalence of (11), (2), and (3) follows directly from $(\dagger)$. To see why (3) is equivalent to (44), recall that the closure of $\varphi(U)$ in $Y$ is the smallest closed subset containing $\varphi(U)$, and so

$$
\varphi(U) \subseteq V \text { if and only if } \overline{\varphi(U)} \subseteq V .
$$

Next, it is also true, for all $U$ in $\mathcal{C}$ losed $(X)$ and $V$ in $\mathcal{C}$ losed $(Y)$, that if $\varphi(U) \subseteq V$ then

$$
U \subseteq \varphi^{-1}(\varphi(U)) \subseteq \varphi^{-1}(V) \subseteq \overline{\varphi^{-1}(V)}
$$

Hence, it follows from the equivalence of (1) and (4) above that $\left(T_{\varphi}, T^{\varphi}\right)$ is an adjoint pair exactly when

$$
U \subseteq \overline{\varphi^{-1}(V)} \Longrightarrow \varphi(U) \subseteq V
$$

for all $U$ in $\mathcal{C l o s e d}(X)$ and $V$ in $\operatorname{Closed}(Y)$.

Now suppose that $\varphi$ is a continuous function. As noted above, $\varphi^{-1}(V)$ is closed in $X$ for all closed subsets $V$ of $Y$, and so

$$
\varphi^{-1}(V)=\overline{\varphi^{-1}(V)}
$$

Therefore, for all $U$ in $\mathcal{C l o s e d}(X)$ and $V$ in $\mathcal{C l o s e d}(Y)$, if

$$
U \subseteq \varphi^{-1}(V)=\overline{\varphi^{-1}(V)}
$$

then

$$
\varphi(U) \subseteq \varphi\left(\varphi^{-1}(V)\right) \subseteq V .
$$

Consequently, $\left(T_{\varphi}, T^{\varphi}\right)$ is an adjoint pair.

Conversely, suppose that $\left(T_{\varphi}, T^{\varphi}\right)$ is an adjoint pair, and fix an arbitrary $V$ in Closed $(Y)$. Set

$$
U:=\overline{\varphi^{-1}(V)}
$$

By $(\ddagger)$

and so

$$
\varphi(U) \subseteq V
$$

$$
\overline{\varphi^{-1}(V)}=U \subseteq \varphi^{-1}(\varphi(U)) \subseteq \varphi^{-1}(V)
$$

Therefore,

$$
\varphi^{-1}(V)=\overline{\varphi^{-1}(V)}
$$

In particular, $\varphi^{-1}(V)$ is closed, and so $\varphi$ is continuous. The theorem follows. 
Acknowledgment. I am grateful to the referees for their helpful comments.

\section{REFERENCES}

[1] B. Coecke and È. O. Paquette, Categories for the practising physicist, in New structures for physics, 173286, Lecture Notes in Phys., 813, Springer, Heidelberg, 2011.

[2] S. Eilenberg and S. MacLane, Natural isomorphisms in group theory, Proc. Nat. Acad. Sci. U. S. A. 28 (1942), 537543.

[3] D. M. Kan, Adjoint functors, Trans. Amer. Math. Soc. 87 (1958), 294-329.

[4] F. W. Lawvere and S. H. Schanuel, Conceptual mathematics: a first introduction to categories, Second edition, Cambridge University Press, Cambridge, 2009.

[5] E. S. Letzter, On continuous and adjoint morphisms between non-commutative spectra, Proc. Ediburgh Math. Soc., 49 (2006), 367-381.

[6] S. Mac Lane, Categories for the working mathematician, Second edition, Graduate Texts in Mathematics, 5, Springer-Verlag, New York, 1998.

[7] B. C. Pierce, Basic category theory for computer scientists, Foundations of Computing Series, MIT Press, Cambridge, MA, 1991.

[8] A. L. Rosenberg, Noncommutative algebraic geometry and representations of quantized algebras, Mathematics and its Applications, 330, Kluwer Academic Publishers Group, Dordrecht, 1995.

[9] S. P. Smith, Maps between non-commutative spaces, Trans. Amer. Math. Soc., 356 (2004), 2927-2944

[10] M. Van den Bergh, Blowing up of non-commutative smooth surfaces, Mem. Amer. Math. Soc., 154 (2001).

Department of Mathematics, Temple University, Philadelphia, PA 19122-6094

E-mail address: letzter@temple.edu 